\title{
Use and misuse of dietary fatty acids for the prevention and treatment of coronary heart disease
}

\author{
Michel DE LORGERIL*, Patricia SALEN
}

LSCPA, UFR de Médecine et Pharmacie, Domaine de la Merci, 38706 La Tronche, France

\begin{abstract}
In the recent years, the health effects of fish (and n-3 fatty acids) have attracted considerable scientific interest. The present consensus is that the cardioprotection of very long chain n-3 fatty acids (also called EPA and DHA) at the low dosage used in recent secondary prevention trials primarily results from an effect on the ischemic myocardium and probably not from an effect on blood lipids and hemostasis. In other words, at these low dosages, there is apparently no major effect of these fatty acids on the progression of the vascular atherosclerotic lesions. In contrast, dietary alpha-linolenic acid (ALA), the parent compound of the very long chain n-3 fatty acids occurring in some vegetable oils, may be protective through mechanisms other than the myocardial (antiarrhythmic) ones. In addition to its own direct preventive effect on cardiac arrhythmias, dietary ALA actually inhibits the elongation and desaturation of linoleic acid (18:2 n-6) into arachidonic acid. Because arachidonic acid (20:4 n-6) plays an important role in inflammation (as the precursor of the proinflammatory eicosanoids and leukotrienes), modifying its amount in blood and cell membranes influences the prevalence and severity of eicosanoid-related disorders, including atherosclerotic complications. The present knowledge of n-3 fatty acids justifies that physicians, in particular cardiologists in the context of secondary prevention of coronary heart disease, manage their patients, the young and the old, to increase their consumption of these fatty acids. They can only advise them to adequately adapt their diet (for instance in primary prevention), but in most cases, the systematic prescription of capsules containing oils enriched in ALA and EPA + DHA will be, ethically and scientifically, an obligation.
\end{abstract}

coronary heart disease / dietary fatty acids / fish / inflammation / sudden cardiac death / atherosclerosis / nutrition

Since Scandinavian investigators suggested that the low mortality rate from coronary heart disease (CHD) among Greenland Eskimos as compared to Europeans might be due to their diet including large quantities of seafood [1], the health effects of fish (and n-3 fatty acids) have attracted considerable scientific interest. Meanwhile, most epidemiological studies have demonstrated a protective effect linked to the con- sumption of even small amounts of fish. The controversy surrounding the association between fish consumption and CHD has, however, been revived by the recent publication of negative results in two large cohort studies in the USA [2,3] and by the inconsistent findings of another two studies $[4,5]$. This apparent inconsistency in results could be due to the following: (1) differences in the methods of dietary assessment

\footnotetext{
* Corresponding author: michel.delorgeril@ujf-grenoble.fr
} 
of fish intake [2]; (2) the different mixes of fish types in the diet, for instance fatty fish versus lean fish, as well as different geographic origins of the fish [5]; (3) the potential contamination of fish by toxic heavy metals in certain areas [6]; (4) a possible bias due to CHD patients (or subjects at high risk of CHD) being aware that fish consumption is cardioprotective, which may result in a reversal of the fish eating-CHD relationship from "cause-effect" to "effect-cause" $[2,3] ;$ (5) differences in the methods used to validate and classify CHD endpoints, with only a few studies reporting, for instance, on both sudden and non-sudden cardiac death; (6) differences in risk levels among different populations, with data suggesting that fish consumption is not associated with a decrease in CHD mortality in low-risk populations whereas it is associated with a markedly reduced CHD mortality in highrisk populations [7]. The last possibility is very simply that the effect of fish and seafood may vary with the amount consumed or, in other words, with the quantity of n-3 fatty acids (the main fatty acids in these foodstuffs) in the diet in relation with the other, saturated and unsaturated, fatty acids.

When assessing more precisely (whenever possible) the cause of cardiac death, however, the epidemiological data suggest that the benefit of eating fish primarily lies in a reduction of sudden cardiac death (SCD). A case-control study in Seattle [8] and a prospective study among US physicians [9] indicate that a modest fish intake may be associated with a $50 \%$ decrease in the risk of SCD but no decrease in the risk of nonsudden cardiac death or myocardial infarction [9]. Moreover, the use of biomarkers (the concentrations of $n-3$ fatty acids) in blood or cells totally confirms the results of studies using the evaluation of dietary intakes [10].

Stronger evidence of an effect of the longchain n-3 fatty acids (Fig. 1), which are relatively abundant in fatty fish (docosahexanoic [DHA] and eicosapentanoic [EPA] acids), on SCD came from laboratory and clinical research indicating that these fatty acids have potent anti-arrhythmic properties, as summarized in a recent article [11]. Alexander Leaf's team, for instance, described the electrophysiological effects of these fatty acids in cultured cardiac myocytes as well as their antiarrhythmic effects in laboratory animals [12]. Billman et al. showed that in a dog model initially used to elucidate the role of the autonomic nervous system on ischemic ventricular fibrillation, intravenous administration of $\mathrm{n}-3$ fatty acids prevented SCD during myocardial ischemia [13]. Christensen et al. reported a randomized trial investigating the effect of longchain n-3 fatty acids on heart rate variability in patients with recent acute myocardial infarction and a low left ventricular ejection fraction [14]. High heart rate variability is thought to reflect high baroreflex sensitivity and has been clearly associated with good outcomes in patients having survived a recent acute coronary event [15]. Christensen et al. have found a positive correlation between n-3 fatty acids in the diet (and blood) and baroreflex sensitivity in their CHD patients [14]. In another study, they showed that the intake of n-3 fatty acids is associated with an increased heart rate variability in a dosedependent manner [16]. The preventive effect of n-3 fatty acids on the risk of SCD is therefore presumably partly related to an anti-arrhythmic effect due to a favorable shift in the vagal/sympathetic balance, an effect known to decrease the susceptibility to ventricular arrhythmias [17]. In addition to an effect on cardiac ion channels [11] and heart rate variability [17], n-3 fatty acids may protect the heart through a third mechanism called preconditioning. In animal models of myocardial, ischemia, dietary fish oil supplementation has been shown to significantly reduce infarct size [18], indicating that the myocardium, whose cells are rich in n-3 fatty acids, is resistant to ischemia. It is likely that this ability to resist to ischemia results from a direct effect of n-3 fatty acids on energy metabolism [19]. Even though the details of the anti-arrhythmic action for n-3 fatty acids remain to be elucidated (the 


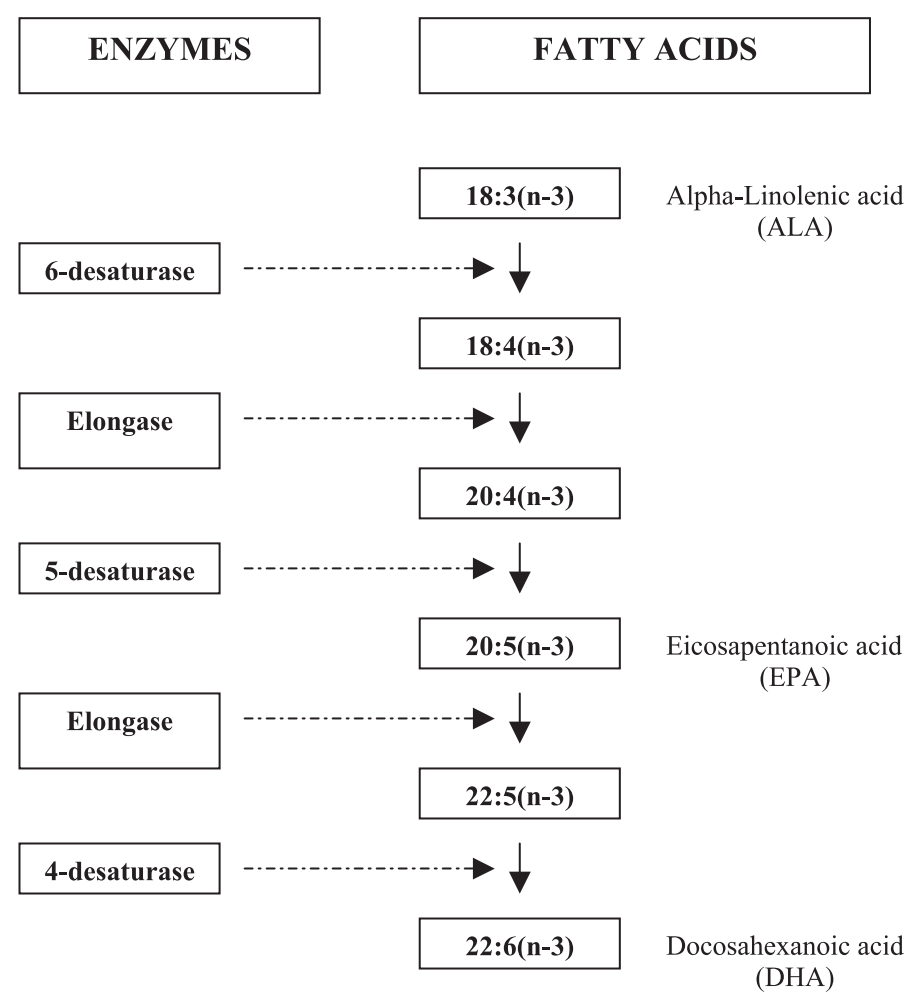

Figure 1. The desaturation and elongation pathway of the n-3 fatty acids, departing from the essential alpha-linolenic acid (ALA) found in some natural vegetable foods, such as walnut and canola oil. Note that, in competition with arachidonic acid, EPA is the starting point for the synthesis of prostaglandins and eicosanoids (see text). Increasing the intake of EPA and DHA, through increased consumption of marine foods, and increasing the intake of ALA are therefore two different preventive strategies in cardiology.

relative importance of the effect on cardiac ion channels, on the autonomic nervous system, on the preconditioning phenomenon, or even on the local production of proarrhythmic and anti-arrhythmic eicosanoids), there is now a large consensus to say that n-3 fatty acids have an important cardioprotective effect in patients with established CHD and that cardiologists should recommend a minimum amount of fish in the diet of their patients [20,21]. In contrast, large amounts have not been shown to be cardioprotective. This is a very important point when considering strategies to prevent CHD. The consensus is also that the cardioprotection of long chain n-3 fatty acids at the low dosage used in the trials primarily results from an effect on the ischemic myocardium and probably not from an effect on blood lipids and hemostasis. In others words, at these low dosages, there is apparently no major effect on the progression of the vascular lesions. In contrast, dietary alpha-linolenic acid (ALA), the parent compound of the long chain n-3 fatty acids (Fig. 1) occurring in some vegetable oils, may be protective through mechanisms other than the myocardial ones (see below).

Indeed, for those who cannot (or will not) eat fish or other seafoods rich in long 
chain n-3 fatty acids, a less direct route to obtain the desirable levels of these fatty acids in their plasma and their cell (in particular cardiac cell) membrane is an increased consumption of ALA (Fig. 1). In addition to its own direct preventive effect on cardiac arrhythmias [12,13], dietary ALA also has the major advantage of never accumulating within cells and of inducing a marked shift in the endogenous metabolism of $n-6$ fatty acids [22]. Dietary ALA actually inhibits the elongation and desaturation of linoleic acid (18:2 n-6) into arachidonic acid [23]. Because arachidonic acid (20:4 n-6), in competition with EPA, plays an important role in inflammation (as the precursor of the proinflammatory eicosanoids and leukotrienes), modifying its amount and the amount of its fatty acid precursors almost certainly influences the prevalence and severity of eicosanoid-related disorders, including atherosclerotic complications and also SCD [22, 23]. As a matter of fact, dietary ALA has been shown to be inversely associated with the risk of fatal CHD [24]. Thus, for many authors, it is the balance between n- 3 and n- 6 fatty acids, rather than the absolute amounts of n-3 fatty acids in the diet, that is critical for prevention [25-27], and the importance of ALA in health and disease is now widely recognized [28, 29].

The underlying theory is that there is no major difference between the dietary and pharmacological application, and that eating fish and taking a capsule of n-3 fatty acids are basically the same thing and should be analyzed in the same way. Accordingly, whatever the way of taking n-3 fatty acids, the biological and physiological effect of the nutrient should be similar to that of the molecule. Can the GISSI trial [30] be consistently compared with the DART intervention [31]? The two trials actually provide similar clinical results but their settings were so different that the interpretation of the data may be different. In GISSI, the patients (all of whom were Italian) were advised to follow a Mediterranean diet. They did so, as shown by the fact that at the end of the trial, more that $82 \%$ of them were regularly using olive oil [30]. In addition, Marchioli and co-workers reported that those patients who most closely adopted Mediterranean dietary habits were best protected [32]. In other words, patients who did not comply with the Mediterranean diet were three times less protected than those who did [32]. However, while the exact interaction between the Mediterranean diet (or some of its characteristics) and n-3 fatty acids remains to be elucidated, the clinical effectiveness of capsules containing $0.8 \mathrm{~g}$ of n-3 fatty acids which was demonstrated in the GISSI trial, was actually observed in patients following a Mediterranean diet with probably a low intake in n-6 fatty acids and a large intake in oleic acid. From a practical point of view, this is very important.

What about the DART investigation? Is it simply a fish (or fish oil) trial? Whereas the trial has often been seen as an experiment testing the effect of a single factor, it is actually a trial investigating the effect of intensive dietary advice on the risk of recurrence in patients with established CHD [31]. In this trial, patients were initially seen in their homes by a nutritionist and randomized into one of three study diets in a factorial design [31]. They were visited again after one month, three months and six months, and thereafter they were contacted at three monthly intervals until two years after the entry into the trial. Those randomized to fish advice were encouraged to eat two portions of fatty fish a week and as much other fish as they could manage. Such an intensive and repeated dietary advice is obviously not just a recommendation to eat fish. When patients eat more fish (fatty fish or lean fish), they do not eat something else, presumably meat, so that they probably reduce their consumption of saturated fatty acids from terrestrial animals. Because of the associated intensive dietary advice, we can speculate that when the patients ate fish, they did not use butter and cream to prepare it, and also that they avoided some forms of preparation (deep fat fried, highly salted fish). In other words, eating more fish is almost necessarily more than a simple increase 
in the intake of n-3 fatty acids. It is also less saturated fatty acids and probably less n-6 fatty acids (those found in the vegetable oils often used to fry fish).

The same reasoning can apply to other dietary trials, for instance the Lyon Diet Heart Study [33] or the Indian Heart trial [34]. In these investigations, patients randomized into the experimental group increased their intake of n-3 fatty acids (specifically ALA in these two trials) through the consumption of foods (essentially canola oil and tree nuts) that also include potentially cardioprotective nutrients (oleic acid, alphatocopherol, folates, arginine, etc.) other than n-3 fatty acids. Moreover, in these trials, patients did decrease their consumption of n-6 fatty acids. It is noteworthy that in the two trials, the rates of both fatal and nonfatal CHD complications were reduced, suggesting that the protective effect of ALA is probably not restricted to a myocardial anti-arrhythmic effect as shown with the long chain n-3 fatty acids [30, 31]. In these food-based trials, however, it is quite difficult to make the difference between the health benefits resulting from n-3 fatty acids (a moderate increase), n- 6 fatty acids (a decrease), from nutrients other than n-3 and $n-6$ fatty acids and from the interaction between the different types of nutrients. There is no room here to discuss each aspect of that complex question.

It remains, however, that we now have strong evidence that increasing the consumption of n-3 fatty acids in CHD patients results in a highly protective effect, in particular against the risk of SCD. In consequence, physicians should manage their patients so that their blood and cell concentrations in n-3 fatty acids should be high. From a practical point of view, they can advise their patients to adequately change their diet. However, in secondary prevention, cardiologists should consider as an obligation to increase the n- 3 fatty acid concentrations in the blood and cell of their high risk patients. For that purpose, the systematic prescription of capsules of oils enriched in both ALA and EPA + DHA is strongly advised. This will soon be recognized as an ethical and medical obligation.

\section{REFERENCES}

[1] Bang HO, Dyerberg J, Sinclair HM. The composition of the Eskimo food in northwestern Greenland. Am J Clin Nutr 1980, 33: 26572661

[2] Ascherio A, Rimm EB, Stampfer MJ, Giovannucci EL, Willett WC. Dietary intake of marine n-3 fatty acids, fish intake, and the risk of coronary heart disease among men. $\mathrm{N}$ Engl J Med 1995, 332: 978-982.

[3] Morris MC, Manson JE, Rosner B, Buring JE, Willett WC, Hennekens CH. Fish consumption and cardiovascular disease in the Physicians' Health Study: a prospective study. Am J Epidemiol 1995, 142: 166-175.

[4] Daviglus ML, Stamler J, Orencia AJ. Fish consumption and the 30-year risk of fatal myocardial infarction. N Engl J Med 1997, 336: 1046-1053.

[5] Oomen CM, Feskens EJ, Räsänen L. Fish consumption and coronary heart disease mortality in Finland, Italy, and the Netherlands. Am J Epidemiol 2000, 151: 999-1006.

[6] Salonen JT, Seppänen K, Nyyssönen K. Intake of mercury from fish, lipid peroxidation, and the risk of myocardial infarction and coronary, cardiovascular, and any death in eastern Finnish men. Circulation 1995, 91: 645-655.

[7] Marckmann P, Gronbaek M. Fish consumption and coronary heart disease mortality. A systematic review of prospective cohort studies. Eur J Clin Nutr 1999, 53: 585-590.

[8] Siscovick DS, Raghunathan TE, King I. Dietary intake and cell membrane levels of longchain omega-3 polyunsaturated fatty acids and the risk of primary cardiac arrest. JAMA 1995, 274: 1363-1367.

[9] Albert CM, Hennekens CH, O’Donnel CJ Fish consumption and risk of sudden cardiac death. JAMA 1998, 279: 23-28.

[10] Albert CM, Campos H, Stampfer MJ, et al Blood levels of long chain omega- 3 fatty acids and the risk of sudden death. N Engl J Med 2002, 346: 1113-1118. 
[11] Leaf A, Kang JX, Xiao YF, et al. Clinical prevention of sudden cardiac death by $n-3$ polyunsaturated fatty acids and mechanism of prevention of arrhythmias by $\mathrm{n}-3$ fish oils. Circulation 2003, 107: 2646-2652.

[12] Kang JX, Leaf A. Antiarrhythmic effects of polyunsaturated fatty acids: recent studies. Circulation 1996, 94: 1774-1780.

[13] Billman GE, Kang JX, Leaf A. Prevention of ischemia-induced ventricular arrhythmias by dietary pure $n-3$ polyunsaturated fatty acids in dogs. Circulation 1999, 99: 2452-2457.

[14] Christensen JH, Gustenhoff P, Korup E. Effect of fish oil on heart rate variability in survivors of myocardial infarction: a double blind randomised controlled trial. BMJ 1996, 312: 677-678.

[15] La Rovere MT, Bigger JT Jr, Marcus FT, for the ATRAMI (Autonomic Tone, and Reflexes after Myocardial Infarction) Investigators. Baroreflex sensitivity and heart-rate variability in prediction of total cardiac mortality after myocardial infarction. Lancet 1998, 351: 478-484.

[16] Christensen JH, Christensen MS, Dyerberg J. Heart rate variability and fatty acid content of blood cell membranes: a dose-response study with n-3 fatty acids. Am J Clin Nutr 1999, 70: 331-337.

[17] Bigger JT Jr, El-Sherif T. Polyunsaturated fatty acids and cardiovascular events. A fish tale. Circulation 2001, 103: 623-625.

[18] Oskarsson HJ, Godwin J, Gunnar RM, et al. Dietary fish oil supplementation reduces myocardial infarct size in a canine model of ischemia and reperfusion. J Am Coll Cardiol 1993, 21: 1280-1285.

[19] Pepe S, McLennan PL. Cardiac membrane fatty acid composition modulates myocardial oxygen consumption and postischemic recovery of contractile function. Circulation 2002, 105: 2303-2308.

[20] Kromhout D. Fish consumption and sudden cardiac death. JAMA 1998, 279: 65-66.

[21] Krauss RM, Eckel RH, Howard B. AHA dietary guidelines: revision 2000: a statement for healthcare professionals from the Nutrition Committee of the American Heart Association. Circulation 2000, 102: 2284-2299.

[22] Lands WE, Morris A, Libelt B. Quantitative effects of dietary polyunsaturated fats on the composition of fatty acids in rat tissues. Lipids 1990, 25: 505-516.
[23] Ross R. Atherosclerosis: an inflammatory disease. N Engl J Med 1999, 340: 115-126.

[24] Hu FB, Stampfer MJ, Manson JE. Dietary intake of alpha-linolenic acid and risk of fatal ischemic heart disease among women. Am J Clin Nutr 1999, 69: 890-897.

[25] Tsimikas S, Reaven PD. The role of dietary fatty acids in lipoprotein oxidation and atherosclerosis. Curr Opin Lipidol 1998, 9: 301307.

[26] Knapp HR, Reilly IA, Alessandrini P, Fitzgerald GA. In vivo indexes of platelet and vascular function during fish oil administration in patients with atherosclerosis. N Engl J Med 1986, 314: 937-942.

[27] De Deckere EA, Korver O, Verschuren PM, Katan MB. Health aspects of fish and omega3 polyunsaturated fatty acids from plant and marine origin. Eur J Clin Nutr 1998, 52: 749_ 753.

[28] Connor WE. Alpha-linolenic acid in health and disease. Am J Clin Nutr 1999, 69: 827828.

[29] De Lorgeril M, Salen P, Laporte F, de Leiris J. Alpha-linolenic acid in the prevention and treatment of coronary heart disease. Eur Heart J 2001, Suppl D: D26-D32.

[30] GISSI-Prevenzione investigators. Dietary supplementation with n-3 polyunsaturated fatty acids and vitamin $\mathrm{E}$ after myocardial infarction: results of the GISSI-Prevenzione trial. Lancet 1999, 354: 447-455.

[31] Burr ML, Fehily AM, Gilbert JF. Effects of changes in fat, fish, and fibre intakes on death and myocardial reinfarction: The Diet And Reinfarction Trial (DART). Lancet 1989 , 334: 757-761.

[32] Marchioli R, Valagussa F, Del Pinto M. Mediterranean dietary habits and risk of death after myocardial infarction. Circulation 2000, 102 (Suppl II): 379

[33] De Lorgeril M, Salen P, Martin JL. Mediterranean diet, traditional risk factors and the rate of cardiovascular complications after myocardial infarction. Final report of the Lyon Diet Heart Study. Circulation 1999, 99: 779-785.

[34] Singh RB, Rastogi SS, Verma R, et al. Randomised controlled trial of cardioprotective diet in patients with recent acute myocardial infarction: results of one year follow-up. BMJ 1992, 304: 1015-1019. 